\section{Treatment of periodic catatonia with olanzapine: a case report}

\author{
Tratamento da catatonia periódica com \\ olanzapina: um relato de caso
}

\section{Dear Editor,}

Catatonia has been described as one of the most enigmatic phenomenon in psychiatry and neurology. ${ }^{1}$ Catatonia is a rare psychomotor syndrome, which can be associated with psychiatric illnesses such as schizophrenia (catatonic subtype) and manicdepressive disorder, as well as with neurological and medical diseases. $^{2}$ Mostly studied by European psychiatrists, periodic catatonia is rare; their usual treatment of choice consisted of electroconvulsive therapy (ECT) or high doses of oral benzodiazepines. Therapeutically, $60-80 \%$ of the acute catatonic patients respond to lorazepam, a GABA-A receptor agonist. ${ }^{3}$ We report a case of periodic catatonia that responded well to olanzapine.

Patient $A$ is a 37-year-old married Caucasian woman, who presented at least five episodes of catatonic stupor along the last 10 years. The first episode occurred in 1995, when the patient presented with crisis of jealousy and paranoid suspiciousness. She complained that the water and food had been poisoned, and believed that someone was watching her movements inside the house through cameras. She reported auditory hallucination and was verbally and physically aggressive toward her family. Her first psychiatric diagnosis was paranoid schizophrenia and treatment was initiated with haloperidol ( $5 \mathrm{mg} /$ day) and clonazepam ( $2 \mathrm{mg} /$ day), with full symptomatic remission after 4 weeks.

During the next episode (1999), she was admitted in a catatonic state, presenting extreme negativism, and mutism for 48 consecutive hours. Tube feeding was required in that occasion. The diagnosis of periodic catatonia was made. Dramatic response was observed with olanzapine (20 mg/day) and clonazepam (1 mg/day), and she was discharged with no psychotic movements or symptoms. Indeed, she relapsed after discharge, and was submitted to 4 ECT sessions. During the follow-up, she showed no psychotic or residual symptoms with complete restitutio ad integrum, and returned to her usual job, maintaining use of olanzapine (20 mg/day).

During her last hospital admission, no evidence of recent drug or alcohol use was found. She had a normal brain CT, EEG, MRI, and blood work-up (including electrolytes, thyroid, liver and renal function test, and blood counts). In the ward, she presented 3 transient episodes of catatonic state, which lasted for a few hours. EEG was performed during one of the catatonic attacks with negative findings. She was discharged on the 40th day of hospitalization, receiving olanzapine $(20 \mathrm{mg} /$ day $)$, with full recovery, without negative or affective symptoms.

Since the physiopathology of periodic catatonia is unknown, therapeutic efforts have been tested on a mostly trial-and-errors basis. Although this patient had previously responded to ECT and to typical antipsychotics, the use of olanzapine could be justified with basis on previous reports of atypical antipsychotic treatment for some types of catatonia.4-6

Atypical neuroleptics such as olanzapine have a broader range of affinity for neuronal receptors than typical neuroleptics, however it is not clear whether atypicals will prove to be a therapeutic option for catatonia in the future. The use of atypical antipsychotics, replacing or in addition to benzodiazepines, ECT or conventional antipsychotics, should be considered as an alternative for patients whose symptoms did not improve with conventional therapy. But it is important to highlight that systematic recommendation of olanzapine for catotonic states in detriment of currently approved therapeutics cannot be made until controlled trials provide further evidence of efficacy.

Carlos Simon Guzman, Viviane Hyun Mi Myung, Yuan Pang Wang Institute of Psychiatry, Universidade de São Paulo (USP), São Paulo (SP), Brazil

Financing: None

Conflict of interests: None

References

1. Lohr JB, Wisniewski AA. Movement Disorders: a Neuropsychiatric Approach. New York, NY: Guilford; 1987.

2. Taylor MA, Fink M. Catatonia in psychiatric classification: a home of its own. Am J Psychiatry. 2003;160(7):1233-41.

3. Bush G, Fink M, Petrides G, Dowling F, Francis A. Catatonia, II: treatment with lorazepam and electroconvulsive therapy. Acta Psychiatr Scand. 1996;93(2):137-43.

4. Northoff G. What catatonia can tell us about "Top-down modulation": a neuropsychiatric hypothesis. Behav Brain Sci. 2002;25(5):555-77.

5. Cook EH, Olson K, Pliskin N. Response of organic catatonia to risperidone. Arch Gen Psychiatry. 1996;53(1):82-3.

6. Cassidy EM, O'Brien M, Osman MF, Finucane J, O'Keane V. Lethal catatonia responding to high dose olanzapine. J Psychopharmacol. $2001 ; 15(4): 302-4$. 